\title{
立体位阻效应导致的苯酚的区域选择性溴化
}

\author{
马献涛* 周坤洁 任梦娟王梦雨 于 静 \\ (信阳师范学院化学化工学院 信阳 464000)
}

\begin{abstract}
摘要 报道了苯酚与廉价易得的氢溴酸的区域选择性溴化反应. 研究发现使用含有较大取代基的亚砜取代广为使用的 二甲亚砜作为氧化剂，可以极大地提高反应的区域选择性，以中等至优秀的收率、高达 99/1 的区域选择性得到预期的 溴代苯酚产物. 该方法可以很容易放大到 $50 \mathrm{mmol}$ 级别, 并且通过萃取与重结晶分离技术即可得到预期的溴代苯酚产 物.

关键词 苯酚; 区域选择性溴化; 氢溴酸; 亚砜; 位阻效应
\end{abstract}

\section{Steric Hindrance Effect Leading to Regioselective Bromination of Phenols with $\mathrm{HBr}$}

\author{
Ma, Xiantao* Zhou, Kunjie $\quad$ Ren, Mengjuan $\quad$ Wang, Mengyu $\quad$ Yu, Jing \\ (College of Chemistry and Chemical Engineering, Xinyang Normal University, Xinyang 464000)
}

\begin{abstract}
A mild and regioselective bromination of phenols with the cheap and easily-available $\mathrm{HBr}$ is developed. By replacing the common used dimethyl sulfoxide (DMSO) with sulfoxides bearing sterically hindered substituents, the desired brominated phenols could be obtained in moderate to high yields with up to 99/1 regioselectivity. This method could be easily scaled up to $50 \mathrm{mmol}$ scale and has the potential to isolate the desired product by simple extraction and recrystallization, showing great practicality of this new method.

Keywords phenol; regioselective bromation; $\mathrm{HBr}$; sulfoxide; steric hindrance effect
\end{abstract}

\section{Introduction}

Bromated phenols are important synthetic intermediates of some natural products and biologically active compounds. ${ }^{[1]}$ While the toxic and corrosive molecular bromine was frequently used in bromination of phenols via electrophilic aromatic substitution (EAS), despite the drawbacks of low regioselectivity and poor functional group tolerance. ${ }^{[2]}$ Then $\mathrm{N}$-bromosuccinimide (NBS) and the analogues were developed as more preferable alternatives and widely used in organic synthesis, however, these brominating reagents are usually expensive. ${ }^{[3]}$

Inspired by the enzyme-catalyzed oxidative halogenation in nature, ${ }^{[4]}$ the slow release of brominating reagent by oxidation of bromide has recently received much attention. ${ }^{[5]} \mathrm{HBr}$ is the byproduct of $\mathrm{Br}_{2}$-based bromination in ton scale every year, which is readily available, inexpen- sive, easy to store and handle. ${ }^{[6]}$ therefore, it would be worthwhile to develop practical methods to utilize $\mathrm{HBr}$ efficiently. The combination of $\mathrm{HBr}$ with an oxidant such as selectfluor, persulfates, hypervalent iodine, $\mathrm{H}_{2} \mathrm{O}_{2}$, air and dimethyl sulfoxide (DMSO), was then developed for oxidative bromination. ${ }^{[5 a, 5 b, 7]}$ However, the use of selectfluor, persulfates, or hypervalent iodine as the oxidant has obvious drawbacks of expensive price and/or generation of many wastes, while the reaction with the greener $\mathrm{H}_{2} \mathrm{O}_{2}$ or air generally required metal catalysts and/or harsh reaction conditions. In contrast, using the cheap and easily available DMSO seems much attractive, due to the advantages of safety, high atom economy, mild and metal-free conditions.

In 2015, Jiao and coworkers ${ }^{[8]}$ found that the use of stoichiometric DMSO as the oxidant instead of the solvent can greatly improve the reaction efficiency and selectivity,

\footnotetext{
* Corresponding author. E-mail: xiantaoma@126.com

Received July 26, 2019; revised August 22, 2019; published online September 5, 2019.

Project supported by the Key Scientific and Technological Project of Henan Province (No. 192102310031), the Scientific Research Project of Henan Province (No. 19B150018), the Nanhu Scholars Program for Young Scholars of Xinyang Normal University and the Young Core Instructor Program of Xinyang Normal University (No. 2018GGJS-05).

河南省科技攻关(No. 192102310031)、河南省高等学校重点科研(No. 19B150018)、信阳师范学院 “南湖学者奖励计划” 青年项目、信阳师范学院青 年骨干教师计划(No. 2018GGJS-05)资助项目.
} 
leading to an efficient and practical halogenation of arenes. However, owing to the negligibly electronic differences between para- and ortho-position of phenols, obtaining high selectivity at para-position of phenols remains challenging at present. ${ }^{[9]}$ Therefore, it is still highly desired to develop a mild, regioselective and waster-free bromination of phenols.

With our continuous interest in regioselective halogenation, ${ }^{[10]}$ we recently observed an unexpected steric hindrance effect from byproduct leading to a mild and regioselective bromination of phenols with TMSBr. ${ }^{[10 \mathrm{~b}]} \mathrm{How}-$ ever, the use of the expensive and water-sensitive TMSBr narrowed the wide applications of this new method. Herein, we wish to report a mild, efficient and regioselective bromination of phenols with the cheap and easily-available $\mathrm{HBr}$, and by replacing the common used DMSO with sulfoxides bearing sterically hindered substituents. The desired brominated phenols could be obtained in high selectivity ( $p / o$ up to 99/1). Notably, this new method could be easily scaled up to $50 \mathrm{mmol}$ scale without reduction in reaction selectivity and has the potential to isolate the desired product and recycle the byproduct thioether by simple extraction and recrystallization. Moreover, water is the sole byproduct, making the method much greener and practical.

\section{Results and discussion}

The mixture of phenol (1a) and $\mathrm{HBr}$ was initially stirred in a solvent of DMSO at $25{ }^{\circ} \mathrm{C}$, and the target $3 \mathbf{a}$ could be obtained in $25 \%$ yield with a poor selectivity of $65 / 35$ (3a/4a) (Table 1, Entry 1). ${ }^{[1]}$ The reaction at $40{ }^{\circ} \mathrm{C}$ gave target 3a in 55\% yield with $p / o$ selectivity of 72/28 (Entry

Table 1 Conditions screening for regioselective bromination of phenol with $\mathrm{HBr}^{a}$

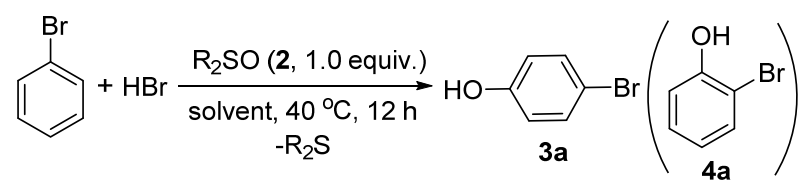

\begin{tabular}{clccc}
\hline Entry & \multicolumn{1}{c}{$\mathrm{R}(\mathbf{2})$} & Solvent & $\mathbf{3 a}^{b} / \%$ & $\mathbf{3 a} / \mathbf{4 a}^{c}$ \\
\hline $1^{d}$ & $\mathrm{Me}(\mathbf{2 a})$ & $\mathrm{DMSO}$ & 25 & $61 / 39$ \\
2 & $\mathrm{Me} \mathrm{(2a)}$ & $\mathrm{DMSO}$ & 55 & $69 / 31$ \\
3 & $\mathrm{Me} \mathrm{(2a)}$ & $\mathrm{MeCN}$ & 80 & $89 / 11$ \\
4 & $n-\mathrm{Bu}(\mathbf{2 b})$ & $\mathrm{MeCN}$ & 75 & $93 / 7$ \\
5 & $\mathrm{Bn}(\mathbf{2 c})$ & $\mathrm{MeCN}$ & 82 & $98 / 2$ \\
6 & $\mathrm{Ph}(\mathbf{2 d})$ & $\mathrm{MeCN}$ & 86 & $95 / 5$ \\
7 & $4-\mathrm{MeC}_{6} \mathrm{H}_{4}(\mathbf{2 e})$ & $\mathrm{MeCN}$ & 87 & $96 / 4$ \\
8 & $4-\mathrm{ClC}_{6} \mathrm{H}_{4}(\mathbf{2 f})$ & $\mathrm{MeCN}$ & 91 & $97 / 3$ \\
9 & $4-\mathrm{ClC}_{6} \mathrm{H}_{4}(\mathbf{2 f})$ & $\mathrm{EtOAc}$ & 84 & $94 / 6$ \\
10 & $4-\mathrm{ClC}_{6} \mathrm{H}_{4}(\mathbf{2 f})$ & $\mathrm{DMF}$ & 0 & - \\
11 & $4-\mathrm{ClC}_{6} \mathrm{H}_{4}(\mathbf{2 f})$ & $\mathrm{CHCl}$ & 41 & $62 / 38$ \\
\hline
\end{tabular}

${ }^{a}$ Unless otherwise noted, a mixture of $1 \mathrm{a}(0.50 \mathrm{mmol}), \mathrm{HBr}(0.525 \mathrm{mmol})$, sulfoxide $2(0.50 \mathrm{mmol})$ and a solvent $(2.0 \mathrm{~mL})$ was directly sealed under air in a Schlenk tube, and stirred at $40{ }^{\circ} \mathrm{C}$ for $12 \mathrm{~h}$, then monitored by thin-layer chromatography (TLC) and/or GC-MS. ${ }^{b}$ Isolated yield based on 1a. ${ }^{c}$ Determined by GC-MS. ${ }^{d} 25{ }^{\circ} \mathrm{C}$.
2). To our delight, using stoichiometric DMSO as the oxidant greatly promoted the reaction, affording the target $\mathbf{3 a}$ in a much higher yield $(80 \%)$ with a higher selectivity of 89/11 (Entry 3). ${ }^{[12]}$ Inspired by our previous regioselective bromination of phenols with TMSBr, ${ }^{[10 \mathrm{~b}]}$ various sulfoxide surrogates such as di-butyl-, di-benzyl-, di-phenyl-, di-4-tolyl- and di-4-chlorphenyl-sulfoxide were then screened to further improve the reaction selectivity (Entries $4 \sim 8$ ). As shown in Table 1 , the reaction with sulfoxide surrogate bearing sterically hindered group, generally gave target 3a in high selectivity (Entries $3 \sim 8) .{ }^{[13]}$ The reaction with di-benzyl sulfoxide gave target 3a in $82 \%$ yield with the highest selectivity (98/2) (Entry 5), while the reaction with di-4-chlorphenyl sulfoxide gave target 3a in $91 \%$ yield with a comparable selectivity (97/3) (Entry 8). Therefore, di-4-chlorphenyl sulfoxide was chosen as the best sulfoxide surrogate. The reaction solvents were finally screened to further improve the reaction yield, but no better results were obtained (Entries 9 11).

With the optimized conditions (Table 1, Entry 8) in hand, various phenols were then tested to extend the scope of the method. As shown in Table 2, like the model reaction, both electron-rich and electron-deficient phenols and even naphthols reacted effectively with $\mathrm{HBr}$, affording the desired products $\mathbf{3 a} \sim \mathbf{3} \mathbf{m}$ in moderate to high yields (Table 2, Entries $1 \sim 14$ ). A series of functional groups such as reactive methoxyl, fluoro, chloro, bromo, aldehyde, and ester groups could be tolerated by this method (Entries 3 8 ). The reactions of ortho- or meta-substituted of phenols sucessfully gave para-brominated products with high selectivity (Entries 2 10). As to para-substituted phenols, ortho-brominated products were obtained in good yields with high selectivity (Entries $11 \sim 13$ ). The method can also be extended to oxidative chlorination with concentrated $\mathrm{HCl}$, but the chlorinated product was obtained with poor p/o selectivity (Entry 15). Unfortunately, the method is not suitable for the synthesis of para-iodinated phenols (Entry 16). Then the method was extended to other electron-rich arenes such as anisole and anilines. To our delight, the target para-brominated products were obtained in moderate to high yields with high selectivity (Entries 17 19).

The potential of this new method in large scale synthesis was also investigated. As shown in Scheme 1, the model reaction of phenol (1a) with $\mathrm{HBr}$ could be easily scaled up in $50 \mathrm{mmol}$ scale, affording the desired product 3a in $84 \%$ isolated yield (7.23 g) only by extraction and recrystallization. Moreover, the byproduct $\mathbf{5 f}$ could be recycled by only extraction, and then be readily oxidized into sulfoxide $\mathbf{2 f}$ with $\mathrm{H}_{2} \mathrm{O}_{2},{ }^{[13]}$ with a totally $>80 \%$ recovery. ${ }^{[12]}$ These experimental results showed great practicality of this new method.

According to our experimental results and the literature reports, ${ }^{[8,10 b, 14]}$ the possible reaction mechanism for this regioselective bromination of phenols is depicted in Scheme 2. $\mathrm{HBr}$ is initially oxidized by sulfoxide 2 to 
Table 2 Regioselective bromination of phenols with $\mathrm{HBr}^{a}$

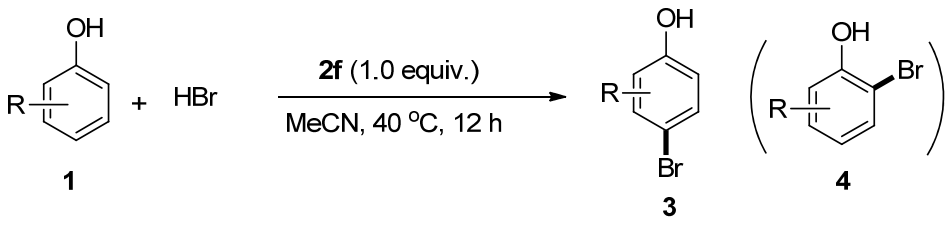<smiles>CCOC(=O)c1cc(Br)ccc1O</smiles>

3h: $81 \%(96 / 4)^{C}$<smiles>Cc1cc(Br)ccc1O</smiles>

3b: $91 \%(99 / 1)^{c}$<smiles>COc1cc(Br)ccc1O</smiles>

3c: $90 \%(95 / 5)^{C}$<smiles>Oc1ccc(Br)cc1F</smiles>

3d: 73\% (99/1)<smiles>Oc1ccc(Br)cc1Cl</smiles>

3e: $84 \%(99 / 1)$<smiles>Oc1ccc(Br)cc1Br</smiles>

3f: $81 \%(92 / 8)$<smiles>O=Cc1cc(Br)ccc1O</smiles>

3g: $74 \%(91 / 9)^{C}$<smiles>Cc1ccc(O)c(Br)c1</smiles>

3j: $73 \%(85 / 15)$<smiles>COc1ccc(O)c(Br)c1</smiles>

I: $85 \%(97 / 3)^{c, d}$<smiles>Oc1ccc(Br)cc1Br</smiles>

3f: $78 \%(99 / 1)^{c, d}$<smiles>Oc1ccc2ccccc2c1Br</smiles>

$3 m: 87 \%(99 / 1)^{e}$

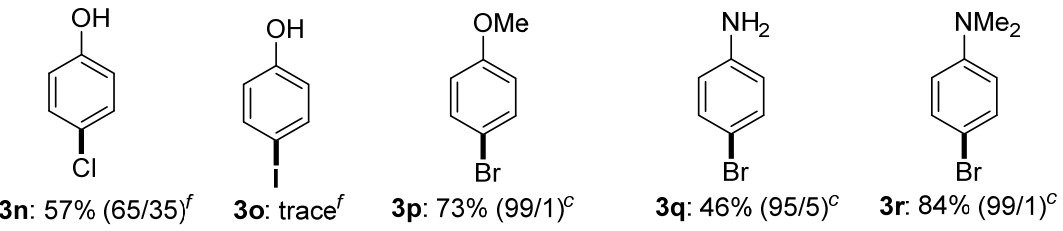

${ }^{a}$ Unless otherwise noted, see Entry 8 of Table 1 for details, isolated yield based on 1, and the ratio of $\mathbf{3} / \mathbf{4}$ in parenthesis was determined by GC-MS; ${ }^{b} 6 \mathrm{~h} ;{ }^{c} 60{ }^{\circ} \mathrm{C}, 12$ $\mathrm{h} ;{ }^{d}$ For $\mathbf{3 k} \sim \mathbf{3 f}$, ortho-brominated products were obtained, the regioselectivity was listed in parenthesis; ${ }^{e} 4 \mathrm{~h} ;{ }^{f} \mathrm{GC}$ yield, $80{ }^{\circ} \mathrm{C}, \mathrm{HX}($ for $\mathbf{3 n}, \mathrm{X}=\mathrm{Cl}$; $\mathbf{3 o}, \mathrm{X}=\mathrm{I})$ was used instead of $\mathrm{HBr}$.

[O] Ref. [15]

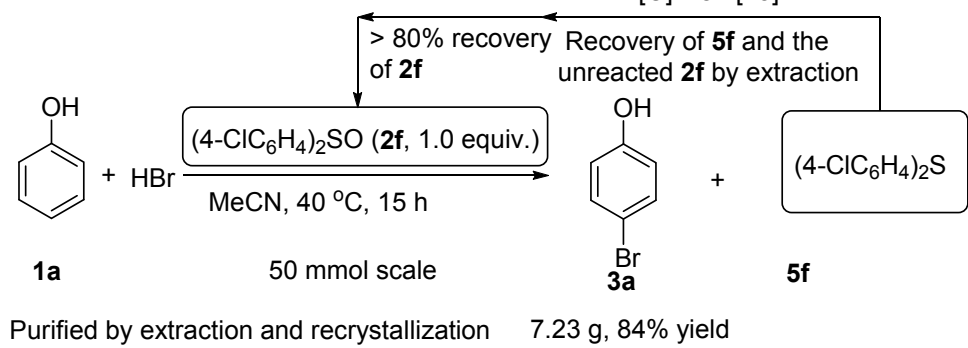

Scheme $150 \mathrm{mmol}$ of scale synthesis of 3a

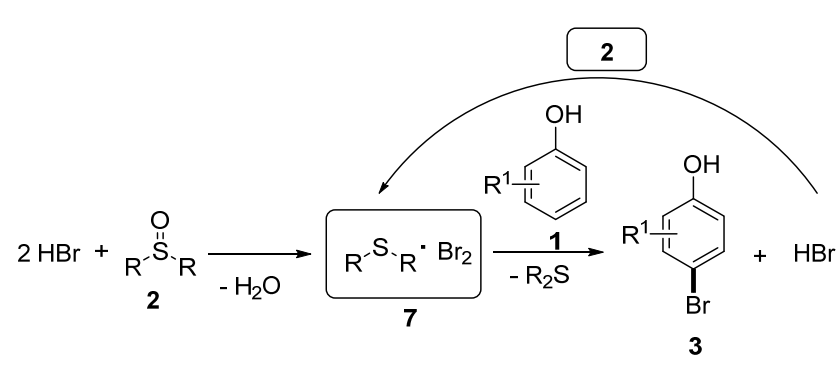

Scheme 2 Possible reaction mechanism

$\mathrm{R}_{2} \mathrm{~S} \cdot \mathrm{Br}_{2}{ }^{[8]}$ Then a EAS bromination of phenols leads to the target product $\mathbf{3}$, meanwhile liberating a molecule of $\mathrm{HBr}$ which could be reoxidized by sulfoxide 2 for the next oxidative cycle. Possibly owing to the steric hindrance effect of $\mathrm{R}$ group of intermediate 7, the electrophilic bromination at the para-position of phenols is much favorable. ${ }^{[10 \mathrm{~b}]}$

\section{Conclusions}

A practical and regioselective bromination of phenols with the cheap and easily-available $\mathrm{HBr}$ was developed. The desired brominated phenols could be obtained with high regioselectivity by replacing the common used DMSO with a sulfoxide bearing sterically hindered substituents. A series of functional groups such as the reactive methoxyl, fluoro, chloro, bromo, aldehyde and ester groups can be tolerated by this method. Moreover, this method could be easily scaled up to $50 \mathrm{mmol}$ and has the potential to isolate the desired product and recycle the byproduct thioether by simple extraction and recry- 
stallization, showing great practicality of this new method. Further application of this novel method for the regioselective functionalization of phenols is under way.

\section{Experimental section}

\subsection{General information}

${ }^{1} \mathrm{H}$ NMR and ${ }^{13} \mathrm{C}$ NMR spectra were recorded on a JNM-ECZ600R/S3 (Jeol, Japan) (600 MHz and $150 \mathrm{MHz}$, respectively) using tetramethylsilane as an internal reference. Mass spectra were measured on an Agilent GC-MS-5890A/5975C Plus spectrometer (EI).

All reactions were conducted in a sealed tube under air atmosphere. All the chemicals were purchased from the Energy, Alfa Aesar, and Tansoole Chemical Reagent Co., and used as received.

\subsection{Experimental}

4.2.1 General procedure for regioselective bromination of phenols with $\mathrm{HBr}$

A mixture of phenol 1a $(47.0 \mathrm{mg}, 0.50 \mathrm{mmol}), \mathrm{HBr}$ ( $w=33 \%$, in HOAc, $130 \mathrm{mg}, 0.525 \mathrm{mmol}$ ), di-4-chlorphenyl-sulfoxide $(135.5 \mathrm{mg}, 0.50 \mathrm{mmol})$ and acetonitrile $(2.0 \mathrm{~mL})$ was directly sealed and stirred in a $20 \mathrm{~mL}$ tube at $40{ }^{\circ} \mathrm{C}$ for $12 \mathrm{~h}$. Then the solvent was evaporated and the residue was purified by silica gel chromatography, eluting with ethyl acetate/petroleum ether $(V / V=0 / 100 \sim 1 / 10)$, to give compound $\mathbf{3 a}$.

4-Bromophenol (3a): Colorless solid. m.p. $65 \sim 66{ }^{\circ} \mathrm{C}$ (lit. $\left.{ }^{[3 \mathrm{~d}]} 66 \sim 68{ }^{\circ} \mathrm{C}\right) ;{ }^{1} \mathrm{H}$ NMR $\left(600 \mathrm{MHz}, \mathrm{CDCl}_{3}\right) \delta: 7.42 \sim$ $7.30(\mathrm{~m}, 2 \mathrm{H}), 6.81 \sim 6.66(\mathrm{~m}, 2 \mathrm{H}), 4.97($ br s, $1 \mathrm{H}) ;{ }^{13} \mathrm{C}$ NMR (150 MHz, $\left.\mathrm{CDCl}_{3}\right) \delta: 154.72,132.56,117.27$, 112.94; MS (EI) $m / z: 174,172,155,143,128,117,93,79$, 74,65 .

4-Bromo-2-methylphenol (3b): Colorless solid. m.p. $62 \sim 63{ }^{\circ} \mathrm{C} \quad\left(\right.$ lit. $\left.^{[15]} 63 \sim 65{ }^{\circ} \mathrm{C}\right) ;{ }^{1} \mathrm{H}$ NMR $(600 \mathrm{MHz}$, $\left.\mathrm{CDCl}_{3}\right) \delta: 7.23(\mathrm{~d}, J=1.8 \mathrm{~Hz}, 1 \mathrm{H}), 7.16(\mathrm{dd}, J=8.4,2.4$ $\mathrm{Hz}, 1 \mathrm{H}), 6.64$ (d, $J=8.4 \mathrm{~Hz}, 1 \mathrm{H}), 4.83$ (br s, 1H), 2.21 (s, $3 \mathrm{H}) ;{ }^{13} \mathrm{C} \mathrm{NMR}\left(150 \mathrm{MHz}, \mathrm{CDCl}_{3}\right) \delta: 153.01,133.61$, 129.82, 126.32, 116.60, 112.61, 15.75; MS (EI) $\mathrm{m} / \mathrm{z}: 188$, 186, 168, 141, 117, 107, 89, 77, 63.

4-Bromo-2-methoxyphenol (3c): ${ }^{[16]}$ Colorless oil. ${ }^{1} \mathrm{H}$ NMR $\left(600 \mathrm{MHz}, \mathrm{CDCl}_{3}\right) \delta: 7.05 \sim 6.88(\mathrm{~m}, 2 \mathrm{H}), 6.79(\mathrm{~d}$, $J=8.4 \mathrm{~Hz}, 1 \mathrm{H}), 5.54$ (br s, $1 \mathrm{H}), 3.87(\mathrm{~s}, 3 \mathrm{H}) ;{ }^{13} \mathrm{C} \mathrm{NMR}$ $\left(150 \mathrm{MHz}, \mathrm{CDCl}_{3}\right) \delta: 147.32,144.91,124.23,115.87$, 114.24, 111.68, 56.23; MS (EI) $m / z: 204,202,189,187$, $161,159,145,143,133,131,117,108,94,79,63$.

4-Bromo-2-fluorophenol (3d): ${ }^{[17]}$ Colorless oil. ${ }^{1} \mathrm{H}$ NMR $\left(600 \mathrm{MHz}, \mathrm{CDCl}_{3}\right) \delta: 7.20(\mathrm{dd}, J=9.6,2.4 \mathrm{~Hz}, 1 \mathrm{H})$, $7.15 \sim 7.07(\mathrm{~m}, 1 \mathrm{H}), 6.87(\mathrm{t}, J=9.0 \mathrm{~Hz}, 1 \mathrm{H}), 5.96$ (br s, $1 \mathrm{H}) ;{ }^{13} \mathrm{C}$ NMR $\left(150 \mathrm{MHz}, \mathrm{CDCl}_{3}\right) \delta: 151.00(\mathrm{~d}, J=242.6$ $\mathrm{Hz}), 142.67$ (d, $J=13.6 \mathrm{~Hz}), 128.05$ (d, $J=3.7 \mathrm{~Hz}), 119.22$ (d, $J=21.4 \mathrm{~Hz}), 118.74$ (s), 111.96 (d, $J=8.3 \mathrm{~Hz}$ ); MS (EI) $m / z: 192,190,172,163,161,144,142,111,95,83$, 63.

4-Bromo-2-chlorophenol (3e): ${ }^{[18]}$ Colorless oil. ${ }^{1} \mathrm{H}$ NMR $\left(600 \mathrm{MHz}, \mathrm{CDCl}_{3}\right) \delta: 7.59$ (d, $\left.J=2.4 \mathrm{~Hz}, 1 \mathrm{H}\right), 7.32$ (dd, $J=8.4,2.4 \mathrm{~Hz} 1 \mathrm{H}), 6.90(\mathrm{~d}, J=8.4 \mathrm{~Hz}, 1 \mathrm{H}), 5.51$ (br s,
$1 \mathrm{H}) ;{ }^{13} \mathrm{C} \mathrm{NMR}\left(150 \mathrm{MHz}, \mathrm{CDCl}_{3}\right) \delta: 150.75,131.52$, 131.40, 120.89, 117.71, 112.40; MS (EI) $\mathrm{m} / z: 210,208$, 206, 179, 177, 172, 170, 153, 144, 142, 127, 117, 99, 91, 73,63

2,4-Dibromophenol (3f): ${ }^{[15]}$ Colorless oil. ${ }^{1} \mathrm{H}$ NMR (600 $\left.\mathrm{MHz} \mathrm{CDCl}_{3}\right) \delta: 7.59(\mathrm{~d}, J=2.4 \mathrm{~Hz}, 1 \mathrm{H}), 7.32(\mathrm{dd}, J=8.4$, $2.4 \mathrm{~Hz}, 1 \mathrm{H}), 6.90(\mathrm{~d}, J=8.4 \mathrm{~Hz}, 1 \mathrm{H}), 5.51(\mathrm{br}, 1 \mathrm{H}) ;{ }^{13} \mathrm{C}$ NMR $\left(150 \mathrm{MHz}, \mathrm{CDCl}_{3}\right) \delta: 151.70,134.13,132.21$, 117.53, 112.73, 110.94; MS (EI) $m / z: 252,251,223,197$, $173,143,117,92,74,63$.

5-Bromo-2-hydroxybenzaldehyde (3g): Colorless solid. m.p. $104 \sim 105{ }^{\circ} \mathrm{C}$ (lit. $\left.{ }^{[19]} 102 \sim 106{ }^{\circ} \mathrm{C}\right) ;{ }^{1} \mathrm{H}$ NMR $(600$ $\mathrm{MHz}, \mathrm{CDCl}_{3}$ ) $\delta: 10.93$ (br s, $\left.1 \mathrm{H}\right), 9.83$ (br s, 1H), 7.67 (d, $J=2.4 \mathrm{~Hz}, 1 \mathrm{H}), 7.59$ (dd, $J=9.0,2.4 \mathrm{~Hz}, 1 \mathrm{H}), 6.90$ (d, $J=$ $9.0 \mathrm{~Hz}, 1 \mathrm{H}) ;{ }^{13} \mathrm{C} \mathrm{NMR}\left(150 \mathrm{MHz}, \mathrm{CDCl}_{3}\right) \delta$ : 195.55, $160.64,139.81,135.73,121.82,119.91,111.46$; MS (EI) $m / z: 202,200,184,182,173,171,145,143,117,107,92$, $77,63$.

Ethyl 5-bromo-2-hydroxybenzoate (3h): ${ }^{[20]}$ Colorless oil. ${ }^{1} \mathrm{H}$ NMR (600 MHz, $\mathrm{CDCl}_{3}$ ) $\delta: 10.79$ (br s, 1H), 7.95 $(\mathrm{d}, J=2.4 \mathrm{~Hz}, 1 \mathrm{H}), 7.51$ (dd, $J=9.0,2.4 \mathrm{~Hz}, 1 \mathrm{H}), 6.87(\mathrm{~d}$, $J=9.0 \mathrm{~Hz}, 1 \mathrm{H}), 4.40(\mathrm{q}, J=7.2 \mathrm{~Hz}, 2 \mathrm{H}), 1.41(\mathrm{t}, J=7.2$ $\mathrm{Hz}, 3 \mathrm{H}) ;{ }^{13} \mathrm{C}$ NMR $\left(150 \mathrm{MHz}, \mathrm{CDCl}_{3}\right) \delta: 169.21,160.73$, $138.39,132.26,119.64,114.17,110.82,62.01,14.25 ; \mathrm{MS}$ (EI) $m / z: 246,244,218,216,200,198,172,170,159,143$, $119,91,81,63$.

4-Bromo-3-methylphenol (3i): $:^{[3 \mathrm{~d}]}$ Colorless solid. m.p. $59 \sim 60{ }^{\circ} \mathrm{C} ;{ }^{1} \mathrm{H}$ NMR $\left(600 \mathrm{MHz}, \mathrm{CDCl}_{3}\right) \delta: 7.33(\mathrm{~d}, J=8.4$ $\mathrm{Hz}, 1 \mathrm{H}), 6.73(\mathrm{~d}, J=3.0 \mathrm{~Hz}, 1 \mathrm{H}), 6.54(\mathrm{dd}, J=8.4,3.0 \mathrm{~Hz}$, 1H), 5.12 (br s, 1H), 2.32 (s, 3H); ${ }^{13} \mathrm{C}$ NMR (150 MHz, $\left.\mathrm{CDCl}_{3}\right) \delta: 154.93,139.19,133.09,117.89,115.37,114.59$, 23.07; MS (EI) $m / z: 188,186,168,141,117,107,89,77$, 63.

3,4-Dibromophenol (3j): ${ }^{[21]}$ Colorless oil. ${ }^{1} \mathrm{H}$ NMR (600 $\left.\mathrm{MHz}, \mathrm{CDCl}_{3}\right) \delta: 7.43(\mathrm{~d}, J=8.4 \mathrm{~Hz}, 1 \mathrm{H}), 7.13$ (d, $J=3.0$ $\mathrm{Hz}, 1 \mathrm{H}), 6.67$ (dd, $J=8.4,3.0 \mathrm{~Hz}, 1 \mathrm{H}), 5.35$ (br s, $1 \mathrm{H}) ;{ }^{13} \mathrm{C}$ NMR $\left(150 \mathrm{MHz}, \mathrm{CDCl}_{3}\right) \delta: 155.41,134.08,125.04$, 120.82, 116.32, 115.35; MS (EI) $m / z: 252,251,223,197$, $173,143,117,92,74,63$.

2-bromo-4-methylphenol (3k): ${ }^{[22]}$ Colorless oil. ${ }^{1} \mathrm{H}$ NMR (600 MHz, $\left.\mathrm{CDCl}_{3}\right) \delta: 7.27(\mathrm{~d}, J=1.2 \mathrm{~Hz}, 1 \mathrm{H}), 7.00$ (dd, $J=8.4,1.2 \mathrm{~Hz}, 1 \mathrm{H}), 6.91(\mathrm{~d}, J=8.4 \mathrm{~Hz}, 1 \mathrm{H}), 5.46$ (br s, 1H), $2.26(\mathrm{~s}, 3 \mathrm{H}) ;{ }^{13} \mathrm{C}$ NMR $\left(150 \mathrm{MHz}, \mathrm{CDCl}_{3}\right) \delta$ : $150.07,132.26,131.54,129.87,115.86,109.92,20.32 ; \mathrm{MS}$ (EI) $m / z: 188,186,168,141,117,107,89,77,63$.

2-Bromo-4-methoxyphenol (31): ${ }^{[23]}$ Colorless oil. ${ }^{1} \mathrm{H}$ NMR (600 MHz, $\left.\mathrm{CDCl}_{3}\right) \delta: 7.00(\mathrm{~d}, J=3.0 \mathrm{~Hz}, 1 \mathrm{H}), 6.93$ $(\mathrm{d}, J=9.0 \mathrm{~Hz}, 1 \mathrm{H}), 6.79$ (dd, $J=9.0,3.0 \mathrm{~Hz}, 1 \mathrm{H}), 5.13$ (br $\mathrm{s}, 1 \mathrm{H}), 3.74(\mathrm{~s}, 3 \mathrm{H}) ;{ }^{13} \mathrm{C} \mathrm{NMR}\left(150 \mathrm{MHz}, \mathrm{CDCl}_{3}\right) \delta$ : $153.85,146.56,116.88,116.42,115.40,110.00,56.06 ; \mathrm{MS}$ (EI) $m / z: 204,202,189,187,161,159,145,143,133,131$, 117, 108, 94, 79, 63.

1-Bromonaphthalen-2-ol (3m): Colorless solid. m.p. $80 \sim 81{ }^{\circ} \mathrm{C} \quad\left(\right.$ lit. $\left.^{[22]} 80 \sim 82{ }^{\circ} \mathrm{C}\right) ;{ }^{1} \mathrm{H}$ NMR $(600 \mathrm{MHz}$, $\left.\mathrm{CDCl}_{3}\right) \delta: 8.02(\mathrm{~d}, J=8.4 \mathrm{~Hz}, 1 \mathrm{H}), 7.78(\mathrm{~d}, J=8.4 \mathrm{~Hz}$, $2 \mathrm{H}), 7.72(\mathrm{~d}, J=8.4 \mathrm{~Hz}, 1 \mathrm{H}), 7.61 \sim 7.52(\mathrm{~m}, 1 \mathrm{H}), 7.41 \sim$ $7.36(\mathrm{~m}, 1 \mathrm{H}), 7.26$ (d, $J=8.4 \mathrm{~Hz}, 1 \mathrm{H}), 5.94$ (br s, $1 \mathrm{H}) ;{ }^{13} \mathrm{C}$ 
NMR $\left(150 \mathrm{MHz}, \mathrm{CDCl}_{3}\right) \quad \delta: 150.67,132.37,129.76$, $129.42,128.31,127.94,125.42,124.23,117.25,106.21$; MS (EI) $m / z: 224,222,195,193,167,143,115,89,74,63$. 1-Bromo-4-methoxybenzene (3o): ${ }^{[22]}$ Colorless oil. ${ }^{1} \mathrm{H}$ NMR $\left(600 \mathrm{MHz}, \mathrm{CDCl}_{3}\right) \delta: 7.52 \sim 7.29(\mathrm{~m}, 2 \mathrm{H}), 6.90 \sim$ $6.66(\mathrm{~m}, 2 \mathrm{H}), 3.77(\mathrm{~s}, 3 \mathrm{H}) ;{ }^{13} \mathrm{C} \mathrm{NMR}\left(150 \mathrm{MHz}, \mathrm{CDCl}_{3}\right) \delta$ : $158.78,132.33,115.82,112.89,55.51$; MS (EI) $m / z: 188$, 186, 173, 171, 145, 143, 129, 117, 107, 92, 77, 63.

4-Bromoaniline (3q): ${ }^{[22]}$ Colorless oil. ${ }^{1} \mathrm{H}$ NMR (600 $\left.\mathrm{MHz}, \mathrm{CDCl}_{3}\right) \delta: 7.24 \sim 7.19(\mathrm{~m}, 2 \mathrm{H}), 6.61 \sim 6.48(\mathrm{~m}, 2 \mathrm{H})$, 3.65 (br, $2 \mathrm{H}) ;{ }^{13} \mathrm{C}$ NMR (150 MHz, $\left.\mathrm{CDCl}_{3}\right) \delta: 145.49$, $132.10,116.79,110.29$; MS (EI) $m / z: 173,171,154,143$, 128, 117, 104, 92, 85, 79, 65, 52.

4-Bromo- $N, N$-dimethylaniline $(3 \mathbf{3 r}):{ }^{[22]}$ Colorless oil. ${ }^{1} \mathrm{H}$ NMR $\left(600 \mathrm{MHz}, \mathrm{CDCl}_{3}\right) \delta: 7.32 \sim 7.27(\mathrm{~m}, 2 \mathrm{H}), 6.60 \sim$ $6.55(\mathrm{~m}, 2 \mathrm{H}), 2.91(\mathrm{~s}, 6 \mathrm{H}) ;{ }^{13} \mathrm{C}$ NMR $\left(150 \mathrm{MHz}, \mathrm{CDCl}_{3}\right) \delta$ : 149.57, 131.76, 114.17, 108.57, 40.68; MS (EI) $m / z: 201$, 200, 199, 198, 185, 184, 183, 182, 168, 155, 141, 128, 118, $104,91,77,63,51$.

\subsubsection{General procedure for large scale synthesis}

To a stirred mixture of phenol (1a) $(4.70 \mathrm{~g}, 50 \mathrm{mmol})$, di-4-chlorphenyl-sulfoxide (2f) (13.50 g, $50 \mathrm{mmol})$ and acetonitrile $(200 \mathrm{~mL}), \mathrm{HBr}(w=33 \%$, in HOAc, $13.0 \mathrm{~g}$, $52.5 \mathrm{mmol}, 1.05$ equiv.) was slowly added at room temperature, then the mixture was allowed to stirred at $40{ }^{\circ} \mathrm{C}$ for $18 \mathrm{~h}$. The solvent was evaporated and the residue was alkalified with $1 \mathrm{~mol} / \mathrm{L}$ sodium hydroxide solution. The mixture was then extracted with ethyl acetate $(100 \mathrm{~mL} \times$ 3 ) and the organic phases were combined, dried with anhydrous sodium sulfate, filtered and ethyl acetate was evaporated by a rotary evaporator to recover di-4-chlorphenylsulfoxide (2f) and di-4-chlorphenyl thioether (5f), which were then oxidized to regenerate di-4-chlorphenyl-sulfoxide (2f) (totally $>80 \%$ recovery of $\mathbf{2 f}$ ). The aqueous phase was then acidized with $1 \mathrm{~mol} / \mathrm{L} \mathrm{HCl}$ to $\mathrm{pH}=1$, then extracted with ethyl acetate $(200 \mathrm{~mL} \times 3)$ and the organic phases were combined, dried with anhydrous sodium sulfate, and filtered, and ethyl acetate was evaporated by a rotary evaporator to give the crude $\mathbf{3 a}(8.05 \mathrm{~g}$, $p / o=97 / 3)$. Finally, the crude 3a was recrystallized with EtOAc/PE $(V: V=10: 1)$ to give the pure product $(7.23$ g, $84 \%$ yield).

Bis(4-chlorophenyl)sulfane (5f): colorless solid; m.p. $93 \sim 94{ }^{\circ} \mathrm{C}$ (lit. $\left.{ }^{[24]} 95 \sim 98{ }^{\circ} \mathrm{C}\right) ;{ }^{1} \mathrm{H}$ NMR $(600 \mathrm{MHz}$, $\left.\mathrm{CDCl}_{3}\right) \delta: 7.29 \sim 7.21(\mathrm{~m}, 8 \mathrm{H}) ;{ }^{13} \mathrm{C} \mathrm{NMR}(150 \mathrm{MHz}$ $\left.\mathrm{CDCl}_{3}\right) \delta: 134.0,133.6,132.4,129.6 ; \mathrm{MS}(\mathrm{EI}) \mathrm{m} / \mathrm{z}: 228$, 213, 195, 185, 171, 153, 143, 141, 128, 117, 102, 91, 77, 65 .

Supporting Information Control experiments and copies of ${ }^{1} \mathrm{H}$ NMR and ${ }^{13} \mathrm{C}$ NMR spectra of products. The Supporting Information is available free of charge via the Internet at http://sioc-journal.cn.

\section{References and note}

[1] Fusetani, N.; Matsunaga, S. Chem. Rev. 1993, 93, 1793. (b) Segraves, E. N.; Shah, R. R.; Segraves, N. L.; Johnson, T. A.; Whitman, S.; Sui, J. K.; Kenyon, V. A.; Cichewicz, R. H.; Crews, P.; Holman, T. R. J. Med. Chem. 2004, 47, 4060.

(c) Akai, S.; Kakiguchi, K.; Nakamura, Y.; Kuriwaki, I.; Dohi, T.; Harada, S.; Kubo, O.; Morita, N.; Kita, Y. Angew. Chem., Int. Ed. 2007, 46, 7458.

(d) Qian, S.; Ma, Y.; Gao, S.; Luo, J. Chin. J. Org. Chem. 2018, 38, 1930 (in Chinese).

(钱少平, 马尧睿, 高姗姗, 骆钧飞, 有机化学, 2018, 38, 1930.)

(e) Zhou, P.; Hou, A.; Wang, Y. Chin. J. Org. Chem. 2018, 38, 156 (in Chinese).

(周鹏飞, 侯爱君, 王洋, 有机化学, 2018, 38, 156.)

[2] For reviews: see: (a) Smith, K.; El-Hitil, G. A. Curr. Org. Synth. 2004, 1, 253.

(b) Saikia, A. J.; Borah, P. P. Chem. Rev. 2016, 116, 6837.

[3] For a review, see: (a) Luo, J.; Xu, X.; Zhao, Y.; Liang, H. Chin. J. Org. Chem. 2017, 37, 2873 (in Chinese).

(骆钧飞, 徐星, 赵延超, 梁洪泽, 有机化学, 2017, 37, 2873.)

For selected recent reports, see: (b) Okada, Y.; Yokozawa, M.; Akiba, M.; Oishi, K.; O-kawa, K.; Akeboshi, T.; Kawamura, Y.; Inokuma, S.; Nakamura, Y.; Nishimura, J. Org. Biomol. Chem. 2003, 1,2506 .

(c) Bovonsombat, P.; Ali, R.; Khan, C.; Leykajarakul, J.; Pla-on, K.; Aphimanchindakul, S.; Pungcharoenpong, N.; Timsuea, N.; Arunrat, A.; Punpongjareorn, N. Tetrahedron 2010, 66, 6928.

(d) Racys, D. T.; Warrilow, C. E.; Pimlott, S. L.; Sutherland, A. Org. Lett. 2015, 17, 4782.

(e) Nishimura, J.; Tang, R.-J.; Milcent, T.; Crousse, B. J. Org. Chem. 2018, 83, 930.

[4] For a review, see: Vaillancourt, F. H.; Yeh, E.; Vosburg, D. A.; Garneau-Tsodikova, S.; Walsh, C. T. Chem. Rev. 2006, 106, 3364.

[5] For reviews, see: (a) Podgoršek, A.; Zupan, M.; Iskra, J. Angew. Chem., Int. Ed. 2009, 48, 8424.

(b) Zhang, G.; Wang, Y.; Ding, C.; Liu, R.; Liang, X. Chin. J. Org. Chem. 2011, 31, 804 (in Chinese).

(张国富, 王涌, 丁成荣, 刘仁华, 梁金氶, 有机化学, 2011，31, 804.)

For selected recent reports, see: (c) Werf, A.; Selander, N. Org. Lett. 2015, 17, 6210.

(d) Satkar, Y.; Ramadoss, V.; Nahide, P. D.; García-Medina, E.; Juárez-Ornelas, K. A.; Alonso-Castro, A. J.; Chávez-Rivera, R.; Jiménez-Halla, J. O. C.; Solorio-Alvarado, C. R. RSC Adv. 2018, 8, 17806.

(e) Sorabad, G. S.; Maddani, M. R. New J. Chem. 2019, 43, 6563.

(f) Walter, C.; Fallows, N.; Kesharwani, T. ACS Omega 2019, 4, 6538 .

(g) Semwal, R.; Ravi, C.; Kumar, R.; Meena, R.; Adimurthy, S. J. Org. Chem. 2019, 84, 792.

(h) Satkar, Y.; Yera-Ledesma, L. F.; Mali, N.; Patil, D.; Navarro-Santos, P.; Segura-Quezada, L. A.; Ramírez-Morales, P. I.; Solorio-Alvarado, C. R. J. Org. Chem. 2019, 84, 4149.

(i) Segura-Quezada, A.; Satkar, Y.; Patil, D.; Mali, N.; Wrobel, K.; González, G.; Zárraga, R.; Ortiz-Alvarado, R.; Solorio-Alvarado, C. R. Tetrahedron Lett. 2019, 60, 1551.

[6] For selected recent reports, see: (a) Mal, K.; Sharma, A.; Maulik, P. R.; Das, I. Chem.-Eur.J. 2013, 20, 662.

(b) Liu, C.; Dai, R.; Yao, G.; Deng, Y. J. Chem. Res. 2014, 38, 593.

(c) Song, S.; Li, X.; Sun, X.; Yuan, Y.; Jiao, N. Green Chem. 2015, 17, 3285.

(d) Karki, M.; Magolan, J. J. Org. Chem. 2015, 80, 3701.

(e) Mal, K.; Kaur, A.; Haque, F.; Das, I. J. Org. Chem. 2015, 80, 640 .

(f) Sorabad, G. S.; Maddani, M. R. New J. Chem. 2019, 43, 6563.

[7] (a) Pandit, P. K.; Gayen, S.; Khamarui, S.; Chatterjee, N.; Maiti, D. K. Chem. Commun. 2011, 47, 6933.

(b) Iskra, J.; Murphree, S. S. Tetrahedron Lett. 2017, 58, 645.

(c) Xin, H.; Yang, S.; An, B.; An, Z. RSC Adv. 2017, 7, 13467.

(d) Tomizuka, A.; Moriyama, K. Adv. Synth. Catal. 2019, 361, 
1447.

(e) Xin, H.; Hu, L.; Yu, J.; Sun, W.; An, Z. Catal. Commun. 2017, 93, 1 .

(f) Kajita, H.; Togni, A. ChemistrySelect 2017, 2, 1117.

(g) Cao, L.; Liu, B.; Liu, W.; Yao, G.; Cheng, Q. Chin. J. Org. Chem. 2011, 31, 2178 (in Chinese).

(曹志凌, 刘冰, 刘玮炜, 姚国伟, 程青芳, 有机化学, 2011，31, 2178.)

[8] Song, S.; Sun, X.; Li, X.; Yuan, Y.; Jiao, N. Org. Lett. 2015, 17, 2886 .

[9] For reviews, see: (a) Huang, Z.; Lumb, J.-P. ACS Catal. 2019, 9, 521.

(b) Chen, Z.; Wang, B.; Zhang, J.; Yu, W.; Liu, Z.; Zhang, Y. Org. Chem. Front. 2015, 2, 1107.

(c) Yanagi, T.; Nogi, K.; Yorimitsu, H. Tetrahedron Lett. 2018, 59, 2951.

[10] (a) Ma, X.-T.; Tian, S.-K. Adv. Synth. Catal. 2013, 355, 337.

(b) Ma, X.; Yu, J.; Jiang, M.; Wang, M.; Tang, L.; Wei, M.; Zhou, Q. Eur.J. Org. Chem. 2019, 4593.

[11] Chauhan and coworkers reported a regioselective bromination of phenol with $\mathrm{HBr}$ at room temperature. The target 4-bromophenol could be obtained in $89 \%$ yield, but no experimental details could be found in the literature, see: Srivastava, S. K.; Chauhan, P. M. S.; Bhaduri, A. P. Chem. Commun. 1996, 2679 for details. We attempted for some times, but the target $\mathbf{3 a}$ was obtained only in low yield by using DMSO as a solvent at room temperature.

[12] Our experimental results are consistent with Jiao's observation, ie the use of stoichiometric DMSO as the oxidant instead of as the solvent can greatly improve the reaction efficiency and selectivity, see Ref. [8].

[13] Kakarla, R.; Dulina, R. G.; Hatzenbuhler, N. T.; Hui, Y. W.; Sofia, M. J. J. Org. Chem. 1996, 61, 8347.

[14] Choudhury, L. H.; Parvin, T.; Khan, A. T. Tetrahedron 2009, 65, 9513.

[15] Ghiaci, M.; Sedaghat, M. E.; Ranjbari, S.; Gil, A. Appl. Catal. A: Gen. 2010, 384, 18.

[16] Mabic, S.; Lepoittevin, J.-P. Tetrahedron Lett. 1995, 36, 1705.

[17] Lou, S.-J.; Chen, Q.; Wang, Y.-F.; Xu, D.-Q.; Du, X.-H.; He, J.-Q.; Mao, Y.-J.; Xu, Z.-Y. ACS Catal. 2015, 5, 2846.

[18] Xiong, X.; Yeung, Y.-Y. ACS Catal. 2018, 8, 4033.

[19] Carrigan, M. D.; Sarapa, D.; Smith, R. C.; Wieland, L. C.; Mohan, R. S. J. Org. Chem. 2002, 67, 1027.

[20] Yang, Y.; Lin, Y.; Rao, Y. Org. Lett. 2012, 14, 2874.

[21] Diemer, V.; Begaud, M.; Leroux, F. R.; Colobert, F. Eur. J. Org. Chem. 2011, 341.

[22] Kajita, H.; Togni, A. ChemistrySelect 2017, 2, 1117.

[23] Kerr, D. J.; Willis, A. C.; Flynn, B. L. Org. Lett. 2004, 6, 457.

[24] Liu, Y.; Kim, J.; Seo, H.; Park, S.; Chae, J. Adv. Synth. Catal. 2015, $357,2205$.

(Zhao, C.) 